\title{
Necessidades de atenção de enfermagem a usuários de Centros de Atenção Psicossocial
}

Nursing care needs for users of the Psychosocial Care Centers

Necesidades de atención de enfermería a usuarios de Centros de Atención Psicosocial

Tássia Ghissoni Pedros0 ${ }^{1}$ io https://orcid.org/ 0000-0003-4446-3835

Luiz Jorge Pedrão ${ }^{1}$ id https://orcid.org/ 0000-0002-0321-0455 Marcia Galan Perroca² io https://orcid.org/ 0000-0003-2931-8429

\section{Como citar:}

Pedroso TG, Pedrão LJ, Perroca MG. Necessidades de atenção de enfermagem a usuários de Centros de Atenção Psicossocial. Acta Paul Enferm. 2021;34:eAPE00823.

DOI

http://dx.doi.org/10.37689/actaape/2021A000823

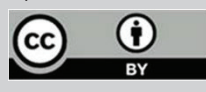

Descritores

Enfermagem psiquiátrica; Centros de Atenção Psicossocial; Avaliação em enfermagem; Cuidados

de enfermagem

Keywords

Psychiatric nursing; Mental health services Nursing assessment; Nursing care

\section{Descriptores}

Enfermería psiquiátrica; Servicios de salud mental; Evaluación en enfermería; Atención de enfermería

Submetido 27 de Abril de 2020

Aceito

20 de Agosto de 2020

\section{Autor correspondente \\ Tássia Ghissoni Pedroso}

E-mail: tassia.gp@gmail.com

\section{Resumo}

Objetivo: Avaliar as necessidades de atenção de enfermagem de usuários de Centros de Atenção Psicossocial; comparar as variáveis demográficas e clínicas entre as categorias de cuidados; e, investigar os fatores preditores para as necessidades de atenção de enfermagem.

Métodos: Estudo descritivo-exploratório com abordagem quantitativa realizado em três Centros de Atenção Psicossocial de uma cidade do no interior do Estado de São Paulo, Brasil. Cinco enfermeiros avaliaram usuários de atendimento intensivo mediante aplicação do Instrumento para Classificação do Nível de Dependência em Enfermagem Psiquiátrica. A análise estatística, com nível de significância de $5 \%(p \leq 0,05)$, consistiu em medidas de tendência central, testes de associação e regressão logística.

Resultados: Nas 249 avalições realizadas houve 145(58,2\%) usuários na categoria de cuidados discreta e $103(41,4 \%)$ na categoria intermediaria. A faixa etária, o número e 0 tipo de diagnósticos estiveram associados às categorias de cuidados: maior frequência da discreta em 30-39 anos, dois/três diagnósticos e transtornos relacionados ao uso de substâncias; e da intermediária/plena em 40-49 anos, um diagnóstico e transtornos psicóticos. Ser usuário de Centros de Atenção Psicossocial II e III, e do sexo masculino foram fatores preditores para maior necessidade de atenção de enfermagem.

Conclusão: Houve predomínio de usuários na categoria discreta e associação entre as categorias de cuidados com faixa etária, número e tipo de diagnósticos. Os fatores de risco para maior necessidade de atenção de enfermagem relacionaram-se aos usuários de Centros de Atenção Psicossocial II e III e do sexo masculino.

\section{Abstract}

Objective: To assess the nursing care needs of users of the Psychosocial Care Centers; compare demographic and clinical variables between care categories; and, investigate the predictive factors for nursing care needs.

Methods: Descriptive-exploratory study with a quantitative approach carried out in three Psychosocial Care Centers in a city in the interior of the State of São Paulo, Brazil. Five nurses assessed intensive care users by applying the Patient Classification Instrument of the Dependency Level in Psychiatric Nursing. The statistical analysis, with a significance level of $5 \%(p \leq 0.05)$, consisted of measures of central tendency, association tests and logistic regression. The statistical analysis, with a significance level of $5 \%(p \leq 0.05)$, consisted of measures of central tendency, association tests and logistic regression.

Results: In the 249 assessments carried out, there were $145(58.2 \%)$ users in the discrete care category and $103(41.4 \%)$ in the intermediate category. The age group, the number and type of diagnoses were associated with the categories of care: higher frequency of the discrete in 30-39 years, two/three diagnoses 
and disorders related to substance use; and intermediate/full in 40-49 years, a diagnosis and psychotic disorders. Being a user of the Psychosocial Care Centers II and III, and male, were predictive factors for greater need for nursing care.

Conclusion: There was a predominance of users in the discrete category and an association between the categories of care with age group, number and type of diagnoses. The risk factors for greater need for nursing care were related to users of the Psychosocial Care Centers II and III and male.

\section{Resumen}

Objetivo: Evaluar las necesidades de atención de enfermería a usuarios de Centros de Atención Psicosocial, comparar las variables demográficas y clínicas entre las categorías de cuidados e investigar los factores predictores de las necesidades de atención de enfermería.

Métodos: Estudio descriptivo y exploratorio, con enfoque cuantitativo realizado en tres Centros de Atención Psicosocial de una ciudad del interior del estado de São Paulo, Brasil. Cinco enfermeros evaluaron usuarios de atención intensiva mediante la implementación del instrumento para la Clasificación del Nivel de Dependencia en Enfermería Psiquiátrica. El análisis estadístico, con nivel de significación de $5 \%(p \leq 0,05)$, consistió en medidas de tendencia central, pruebas de asociación y regresión logística.

Resultados: De las 249 evaluaciones realizadas, 145 (58,2 \%) usuarios se encontraban en la categoría de cuidados discreta y 103 (41,4 \%) en la categoría intermedia. El grupo de edad, el número y el tipo de diagnóstico estuvieron relacionados con las categorías de cuidados: mayor frecuencia en categoría discreta personas entre 30-39 años, dos/tres diagnósticos y trastornos relacionados con el uso de sustancias, y en la intermedia/plena personas entre 40-49 años, un diagnóstico y trastornos psicóticos. Ser usuario de Centros de Atención Psicosocial II y III y ser de sexo masculino fueron factores predictores de una mayor necesidad de atención de enfermería.

Conclusión: Se observó un predominio de usuarios en la categoría discreta y relación entre las categorías de cuidados con el grupo de edad, número y tipo de diagnóstico. Los factores de riesgo de mayor necesidad de atención de enfermería se relacionaron con los usuarios de Centros de Atención Psicosocial II y III y de sexo masculino.

\section{Introdução}

Os movimentos de reforma psiquiátrica mundiais a partir da década de 1960, e no Brasil, em 70, trouxeram novas práticas em saúde mental. ${ }^{(1)}$ A principal estratégia, neste novo cenário foi o surgimento de serviços comunitários substitutivos ao modelo hospitalocêntrico - os Centros de Atenção Psicossocial (CAPS) - para o acolhimento de pessoas com transtornos mentais estimulando sua integração social e familiar e proporcionando apoio na busca por autonomia. ${ }^{(2)}$ Estes centros se diferenciam em modalidade e complexidade de tratamento, clientela atendida e área de abrangência no território. ${ }^{(3)}$

No modelo de atenção desinstitucionalizado, prioriza-se a produção de cuidado integral e a humanização da assistência com foco nas preferências e valores dos usuários. ${ }^{(4,5)} \mathrm{Na}$ área de enfermagem psiquiátrica e saúde mental as discussôes têm avançado acerca do cuidado centrado na pessoa, destacando as atitudes de respeito às suas experiências e necessidades, e envolvimento no plano de tratamento. ${ }^{(6,7)}$

Seguindo essa abordagem, os CAPS implementaram o Projeto Terapêutico Singular (PTS) que estabelece um plano de tratamento construído em conjunto com a equipe interdisciplinar, o próprio usuário e sua família. Nele há valorização do conhecimento e opiniáo do cliente utilizando-se de recursos como a escuta ativa e a educação em saúde, e não se restringindo a terapêutica medicamentosa. As reavaliaçóes fazem parte deste plano de tratamento já que as necessidades podem variar ao longo do período. ${ }^{(3,4)}$

Neste contexto de prática, a atuação da equipe de enfermagem na assistência psiquiátrica e saúde mental estende-se a oferecer um ambiente de acolhimento assim como estabelecer um vínculo de confiança embasado na empatia e no respeito. ${ }^{(8)}$ Portanto, no PTS, a enfermagem destaca-se pela sua proximidade e preocupação em prestar assistência a partir das necessidades de atenção da clientela. ${ }^{(9)}$

Os termos necessidades de atenção, demanda de cuidados ou necessidade de cuidados, vêm sendo empregados e associados aos Instrumentos de Classificação de Pacientes (ICP), cujo objetivo é a identificação do quanto o paciente necessita da atenção de enfermagem em determinado serviço de saúde. ${ }^{(10,11)}$

A literatura cientifica tem disponibilizado estudos abordando o perfil sociodemográfico ${ }^{(12,13)}$ e clínico $^{(14,15)}$ de pessoas assistidas nos CAPS. No entanto, não foram encontradas pesquisas referentes a demanda assistencial destes em relação à enfermagem mediante uso de instrumento de classificação de pacientes. Diante desse cenário, o presente estudo foi guiado pelas seguintes questóes: Quais são as necessidades de atenção de usuários de CAPS? Encontram-se diferenças entre as modalidades? 
Existe associação entre as categorias de cuidados e as variáveis demográficas e clinicas estudadas? Há fatores preditores para maior necessidade de atenção de enfermagem? Dessa forma, seus objetivos constituíram-se em: avaliar as necessidades de atenção de enfermagem a usuários de Centros de Atenção Psicossocial (CAPS); comparar as variáveis demográficas e clínicas entre as categorias de cuidados; e, investigar os fatores preditores para as necessidades de atenção de enfermagem.

\section{Métodos}

Esta investigação de caráter descritivo-exploratória, transversal, com abordagem quantitativa foi realizada no período de março a maio de 2019 em três CAPS, descritos a seguir, destinados ao atendimento a adultos, localizados em uma cidade do interior do Estado de São Paulo, Brasil.

Constituíram-se cenários do estudo os CAPS Álcool e Drogas (CAPSAD), CAPSII e CAPSIII. O CAPSAD oferece atendimento a dependentes de substâncias psicoativas por demanda espontânea ou encaminhados por serviços de saúde abrangendo, na região oeste, uma população estimada em 200.000 habitantes e 1575 atendimentos/mês. O CAPSII, com o distrito central como área de abrangência (estimativa de 120.000 habitantes e 1350 atendimentos/mês), fornece serviços de acolhimento ambulatorial e de atendimento-dia (não intensivos, semi-intensivos e intensivos) em saúde mental. ${ }^{(14)}$ Já o CAPSIII, com funcionamento de 24 horas, realiza consultas ambulatoriais, atendimento-dia e acolhimento noturno, com cinco leitos de permanência por sete dias contínuos ou dez dias descontinuados, com média de 1756 atendimentos/mês.

A população constituiu-se em todos os usuários em atendimento intensivo presentes no período de coleta de dados. Atendimento intensivo caracteriza-se por uma atenção contínua, é oferecido diariamente às pessoas em quadros graves de sofrimento psíquico, em situação de crise ou dificuldades intensas no convívio social e familiar. ${ }^{(16)}$ Esta escolha decorreu da frequência ao serviço (três a quatro/ semana), tempo de permanência (mínimo de seis horas/ dia) e quantidade de procedimentos (25/usuário/ mês), ${ }^{(16)}$ possibilitando aplicação de instrumento de classificação de pacientes. No período da coleta de dados registrou-se a presença de 50 usuários no CAPSII, 60 no CAPSIII e 40 no CAPSAD. Destes, 63 estavam em atendimento intensivo, sendo 20 no CAPSII, 21 no CAPSIII e 22 no CAPSAD.

Investigou-se variáveis demográficas (sexo e idade) e clinicas (número e tipo de diagnósticos psiquiátricos). Devido à existência de até três diagnósticos de alguns usuários, constatado em prontuário, optou-se por considerar, como principal, aquele que gera maior impacto na vida da pessoa segundo literatura científica, considerando estigma, prejuízo social e convívio familiar. ${ }^{(17)}$ No caso do CAPSAD optou-se pelo diagnóstico relacionado ao uso de substâncias. Eles foram agrupados em: 1. Transtornos de humor, 2. Transtornos psicóticos, 3. Transtornos mentais e comportamentais relacionados ao uso de substâncias e, 4. Outras morbidades (Transtornos: de personalidade, de desenvolvimento, de ansiedade, relacionados ao estresse, dissociativo e mental não especificado).

Para avaliação das necessidades de cuidados dos usuários aplicou-se o Instrumento para Classificação do Nível de Dependência em Enfermagem Psiquiátrica (ICP- Enfermagem Psiquiátrica) construído e validado no Brasil. ${ }^{(18)}$ Esta escala encontra-se constituída por 11 áreas de cuidados: Cuidados com a aparência e higiene; Expressão do pensamento; Humor e afeto; Atividades; Interação social; Alimentação e hidratação; Sono; Medicação; Eliminaçóes; Sinais vitais e outros controles; Queixas e problemas somáticos. A pontuação total varia de 11 a 33 e o usuário pode ser classificado em uma de três categorias de cuidados: dependência discreta (11 a 18), dependência intermediária (19 a 26) e dependência plena (27 a 33). Neste estudo, obteve-se alta consistência interna da escala, $\operatorname{com} \alpha$ de Cronbach igual a 0,87 .

Todos os enfermeiros com jornadas de trabalho no período diurno e assistência direta à população do estudo aceitaram participar do estudo, em um total de cinco, e aplicaram o instrumento semanalmente, durante quatro semanas. Devido à dinâmica do serviço, os usuários tiveram avaliaçōes múlti- 
plas. Um dos pesquisadores ficou responsável pela entrega e recolhimento semanal de cópias do ICPEnfermagem Psiquiátrica.

Uma vez que o instrumento não era utilizado nos serviços, o mesmo pesquisador implementou ação educativa junto aos profissionais para propiciar aproximação do seu conteúdo e forma de operacionalização. A atividade foi previamente agendada nos locais de estudo, com duração de 120 minutos. A prática educativa contou com uma situação simulada utilizando casos clínicos do ambiente de prática para verificar se todos entendiam o instrumento da mesma forma. A partir das discussóes constatou-se alta concordância entre os enfermeiros a respeito dos casos clínicos avaliados.

Os dados obtidos foram organizados em planilha do Excel, com dupla digitação. Para todas as análises, processadas nos programas IBM SPSS Statistics versão 25, R i386 versão 3.4 .0 e SAS System for Windows versão 9.2, adotou-se nível de significância de $\mathrm{p} \leq 0,05$. Depois de codificados e tabulados, realizou-se: estatística descritiva e medidas de tendência central (média, mediana e variação); associação entre as variáveis empregando-se o teste Exato de Fisher e Qui-quadrado, e quando não houve normalidade, Mann Whitney e Kruskal-Wallis; estudo dos fatores associados com as categoria de cuidados utilizando a análise de regressão logística dicotômica com modelos univariado e multivariado (com critério Stepwise Forward) e método de odds proporcionais. $\mathrm{Na}$ análise multivariada foram consideradas todas as variáveis da univariada, mesmo as com valor de $\mathrm{p}$ maior que 0,05. Considerou-se que algumas variáveis que não são significativas na univariada podem vir a ser significativas na multivariada na presença de outras variáveis que foram anteriormente selecionadas. As categorias intermediária e plena foram agrupadas para maior consistência nas análises, devido à frequência muito baixa a desta última categoria.

O estudo seguiu as determinaçóes para pesquisas em seres humanos com apreciação e aprovação do Comitê de Ética em Pesquisa da Escola de Enfermagem de Ribeirão Preto da Universidade de São Paulo (CEP/EERP/USP) sob parecer $n^{\circ}$ 3.076.217/2018.

\section{Resultados}

Os cinco enfermeiros avaliadores apresentaram idade média de $44(\mathrm{Dp}=8,5)$ anos; quatro eram do sexo feminino e quatro concluíram pós-graduação (lato-sensu ou stricto senso). Todos possuíam apenas um vínculo empregatício e o tempo de trabalho em CAPS variou de cinco a 13 anos.

Obteve-se 249 avaliaçóes de 63 usuários com $145(58,2 \%)$ na categoria de cuidados discreta, sendo $74(51 \%)$ deles no CAPSAD, e $103(41,4 \%)$ na categoria intermediaria, com 93(90,3\%) nos CAPSII e III. A idade média variou de 44,7 $(\mathrm{D} p=12,4$; CAPSAD) a $52(\mathrm{D} p=10,8$; CAPSIII $)$ anos. Encontrou-se diferença estatisticamente significativa para: sexo ( $\mathrm{p} \leq 0,05$; CAPSIII feminino ₹ CAPSAD e II masculino), diagnostico principal ( $p \leq 0,01$; CAPSII e III Transtornos Psicóticos ₹ CAPSAD Transtornos relacionados ao uso de substâncias) e categorias de cuidados ( $\mathrm{p} \leq 0,01$; CAPSAD discreta ₹ CAPSII e III intermediária) (Tabela 1). No que se refere ao número de diagnósticos psiquiátricos 146(58,6\%) usuários apresentaram um, $72(28,9 \%)$ dois e $31(12,45 \%)$ três diagnósticos.

Considerando-se todos os CAPS, os valores médios dos escores variaram de 1,1(Dp=0,4; CAPSAD, Eliminaçôes) a 2,2 (Dp=0,6; CAPS III, Sinais vitais e outros controles). As áreas de cuidado que apresentaram maiores pontuaçóes foram: expressão do pensamento, atividades, interação social, medicação e sinais vitais e outros controles (CAPSAD); queixas e problemas somáticos, expressão do pensamento, humor e afeto, sinais e outros controles (CAPSII) e sinais e outros controles, humor e afeto, e sono (CAPSIII) (Tabela 2).

Evidenciou-se diferença significativa entre a demanda de atenção em relação à enfermagem (categorias de cuidados discreta, intermediária/plena) com maiores frequências nas variáveis: faixa etária (30-39 anos na categoria discreta; 40-49 anos na intermediária/plena - $\mathrm{p} \leq 0,05$ ); número de diagnóstico (dois e três na categoria discreta e de um na intermediária/plena - $\mathrm{p} \leq 0,01$ ); tipos de diagnósticos (transtornos relacionados ao uso de substâncias na discreta e de transtornos psicóticos na intermediá- 
Tabela 1. Associação entre as variáveis demográficas e clinicas dos usuários e os CAPS investigados

\begin{tabular}{|c|c|c|c|c|c|}
\hline Variáveis & $\begin{array}{c}\text { CAPS AD } \\
\mathrm{n}(\%)\end{array}$ & $\begin{array}{c}\text { CAPS II } \\
\mathrm{n}(\%)\end{array}$ & $\begin{array}{c}\text { CAPS III } \\
\text { n(\%) }\end{array}$ & $\begin{array}{l}\text { Total } \\
\mathrm{n}(\%)\end{array}$ & $p$-value \\
\hline Usuários $(\mathrm{n}=63)$ & $22(34,9)$ & $20(31,7)$ & $21(33,4)$ & & \\
\hline \multicolumn{6}{|l|}{ Sexo } \\
\hline $\begin{array}{l}\text { Masculino } \\
\text { Feminino }\end{array}$ & $\begin{array}{c}18(28,6) \\
4(6,3)\end{array}$ & $\begin{array}{c}11(17,4) \\
9(14,3)\end{array}$ & $\begin{array}{l}10(15,9) \\
11(17,5)\end{array}$ & $\begin{array}{l}39(61,9) \\
24(38,1)\end{array}$ & $\leq 0,05^{\star}$ \\
\hline $\begin{array}{l}\text { Faixa etária (anos) } \\
\begin{array}{l}19-29 \\
30-39 \\
40-49 \\
\geq 50\end{array}\end{array}$ & $\begin{array}{c}3(4,8) \\
5(7,9) \\
3(4,8) \\
11(17,5)\end{array}$ & $\begin{array}{l}1(1,6) \\
2(3,1) \\
8(12,7) \\
9(14,3)\end{array}$ & $\begin{array}{c}1(1,6) \\
2(3,1) \\
3(4,8) \\
15(23,8)\end{array}$ & $\begin{array}{c}5(7,9) \\
9(14,3) \\
14(22,3) \\
35(55,5)\end{array}$ & $\mathrm{NS}^{+}$ \\
\hline $\begin{array}{l}\text { Idade (anos) } \\
\text { Media (DP) } \\
\text { Variaçãa }\end{array}$ & $\begin{array}{c}44,7( \pm 12,4) \\
19,8-60,3\end{array}$ & $\begin{array}{c}49,5( \pm 9) \\
24,5-62,6\end{array}$ & $\begin{array}{l}52( \pm 10,8) \\
25-68,3\end{array}$ & & $\mathrm{NS}^{+}$ \\
\hline $\begin{array}{l}\text { Diagnóstico Principal } \\
\text { Transtornos de Humor } \\
\text { Transtornos Psicóticos } \\
\text { Transtornos Relacionados ao uso de Substâncias } \\
\text { Outras morbidades }\end{array}$ & $\begin{array}{c}- \\
- \\
22(34,9) \\
-\end{array}$ & $\begin{array}{c}2(3,1) \\
14(22,3) \\
1(1,6) \\
3(4,8)\end{array}$ & $\begin{array}{c}6(9,5) \\
10(15,9) \\
- \\
5(7,9)\end{array}$ & $\begin{array}{c}8(12,7) \\
24(38,1) \\
23(36,5) \\
8(12,7)\end{array}$ & $\leq 0,01^{*}$ \\
\hline Avaliações ( $N=249$ ) & $85(34,2)$ & $80(32,1)$ & $84(33,7)$ & & \\
\hline Categorias & & & & & $\leq 0,01^{\ddagger}$ \\
\hline Discreta & $74(29,7)$ & $32(12,8)$ & $39(15,7)$ & $145(58,2)$ & \\
\hline Intermediaria & $10(4,0)$ & $48(19,3)$ & $45(18,1)$ & $103(41,4)$ & \\
\hline Plena & $1(0,4)$ & - & - & $1(0,4)$ & \\
\hline Escores & & & & & $\leq 0,0^{1} \S$ \\
\hline$M(D p)$ & $14,4(3,6)$ & $18,7(3,2)$ & $18,7(3,5)$ & & \\
\hline Md (Q1; Q3) & $14(11,5 ; 16)$ & $19,5(16 ; 21,8)$ & $19(17 ; 21)$ & & \\
\hline Variação & $11-27$ & $12-23$ & $12-25$ & & \\
\hline
\end{tabular}

${ }^{\star}$ Teste Exato de Fischer; †Não significativo; ${ }^{\circ}$ Teste Qui-Quadrado de Pearson; 8 Kruskal-Wallis; DP - desvio padrão; M - média; Md - mediana; Q1 - quartil 1; 03 - quartil 3

Tabela 2. Escores médios das áreas de cuidados do instrumento de classificação por CAPS investigada $(n=249)$

\begin{tabular}{|c|c|c|c|c|c|c|}
\hline \multirow{2}{*}{$\begin{array}{l}\text { Áreas de } \\
\text { Cuidados }\end{array}$} & \multicolumn{2}{|c|}{ CAPS AD $(n=85)$} & \multicolumn{2}{|c|}{ CAPS II $(n=80)$} & \multicolumn{2}{|c|}{ CAPS III $(n=84)$} \\
\hline & $M(D P)$ & $\operatorname{Md}(Q 1: Q 3)$ & $M(D P)$ & $\operatorname{Md}(Q 1: Q 3)$ & $M(D P)$ & $\operatorname{Md}(Q 1: Q 3)$ \\
\hline AC1 & $1,2(0,5)$ & $1(1: 1)$ & $1,3(0,6)$ & $1(1: 2)$ & $1,7(0,7)$ & $2(1: 2)$ \\
\hline AC2 & $1,5(0,6)$ & $1(1: 2)$ & $1,9(0,7)$ & $2(1: 2)$ & $1,7(0,7)$ & $2(1: 2)$ \\
\hline AC3 & $1,3(0,6)$ & $1(1: 2)$ & $1,9(0,5)$ & $2(2: 2)$ & $1,9(0,6)$ & $2(1: 2)$ \\
\hline AC4 & $1,4(0,6)$ & $1(1: 2)$ & $1,6(0,6)$ & 2(1:2) & $1,7(0,6)$ & $2(1: 2)$ \\
\hline AC5 & $1,4(0,5)$ & 1(1:2) & $1,6(0,5)$ & $2(1: 2)$ & $1,7(0,6)$ & $2(1: 2)$ \\
\hline AC6 & $1,2(0,4)$ & $1(1: 1)$ & $1,6(0,5)$ & $2(1: 2)$ & $1,3(0,5)$ & $1(1: 2)$ \\
\hline AC7 & $1,2(0,4)$ & $1(1: 1)$ & $1,7(0,4)$ & $2(1,2: 2)$ & $1,9(0,5)$ & $2(2: 2)$ \\
\hline AC8 & $1,4(0,5)$ & $1(1: 2)$ & $1,8(0,4)$ & 2(2:2) & $1,5(0,5)$ & 2(1:2) \\
\hline AC9 & $1,1(0,4)$ & $1(1: 1)$ & $1,1(0,3)$ & $1(1: 1)$ & $1,2(0,4)$ & $1(1: 1)$ \\
\hline AC10 & $1,4(0,8)$ & $1(1: 1)$ & $1,9(0,7)$ & 2(1:2) & $2,2(0,6)$ & $2(2: 3)$ \\
\hline AC11 & $1,3(0,7)$ & $1(1: 1)$ & $2,1(0,3)$ & $2(2: 2)$ & $1,8(0,6)$ & $2(1: 2)$ \\
\hline
\end{tabular}

AC1 - Cuidados com Aparência e Higiene; AC2 - Expressão do Pensamento; AC3 - Humor e Afeto; $A C 4$ - Atividades; AC5 - Interação Social; AC6 -Alimentação/Hidratação; AC7 - Sono; AC8 - Medicação; AC9 Eliminações; AC10 - Sinais vitais e outros controles; AC11 - Queixas e problemas somáticos; M - média; DP - desvio padrão; Md - mediana; 01 - quartil 1; Q3 - quartil 3

ria/plena; e modalidades CAPS (categoria discreta na $\mathrm{AD}$ e intermediaria/plena nos CAPS II e III).

A partir da análise de regressão logística dicotômica, descrita na tabela 3, verificou-se que as variáveis CAPS e sexo estiveram significativamente associadas a uma maior demanda de atençáo de enfermagem. Os usuários com chances elevadas constituíram-se em CAPS II e III, com riscos 16,4 e 13,6 vezes maior em serem classificados na categoria intermediária/plena, respectivamente; e os do sexo masculino, com probabilidade de 3,8 vezes.

\section{Discussão}

A periodicidade das avaliaçóes pode constituir em uma limitação por ter sido realizada semanalmente e não de acordo com a frequência de comparecimento dos usuários em atendimento intensivo, de três a cinco vezes por semana. Ainda, por representar os CAPS de uma cidade no interior do estado de Sáo Paulo, os achados podem diferir de outros cenários.

Tratou-se de uma investigação pioneira por ser a primeira aproximação de enfermeiros da Atenção à Saúde Mental Comunitária com uma ferramenta de avaliação da demanda de cuidados de enfermagem. Com isso, foi possível obter um mapeamento do perfil assistencial de usuários em diferentes modalidades de CAPS, associou-se às suas características demográficas e clínicas, permitindo, nortear o planejamento dos cuidados de enfermagem na construção do PTS.

Neste estudo o ICP - Enfermagem Psiquiátrica foi aplicado para avaliar as necessidades de atenção 
Tabela 3. Análise de regressão logística univariada e multivariada entre dois grupos de demanda de atenção de enfermagem (categorias de cuidados discreta vs intermediária/ plena) $(n=249)$

\begin{tabular}{|c|c|c|c|c|c|c|}
\hline \multirow{2}{*}{ Variáveis } & \multicolumn{3}{|c|}{ Análise univariada } & \multicolumn{3}{|c|}{ Análise multivariada } \\
\hline & OR & IC $95 \%$ & $p$-value & OR & IC $95 \%$ & $p$-value \\
\hline \multicolumn{7}{|l|}{ Sexo } \\
\hline Feminino & 1 & - & - & 1 & - & \\
\hline Masculino & 1,61 & $\begin{array}{l}0,95- \\
2,73\end{array}$ & 0,08 & 3,79 & $\begin{array}{c}2,01 \\
-7,14\end{array}$ & $<0,001$ \\
\hline \multicolumn{7}{|l|}{ Faixa Etária } \\
\hline 19-29 anos & 1 & - & - & - & - & - \\
\hline 30-39 anos & 0,42 & $\begin{array}{c}0.13- \\
1.35\end{array}$ & 0,15 & - & - & - \\
\hline 40-49 anos & 1,63 & $\begin{array}{c}0.58- \\
4.55\end{array}$ & 0,35 & - & - & - \\
\hline$\geq 50$ anos & 0,80 & $\begin{array}{c}0.31- \\
2.02\end{array}$ & 0,62 & - & - & - \\
\hline \multicolumn{7}{|l|}{ Unidade CAPS } \\
\hline CAPSAD & 1 & - & - & 1 & - & - \\
\hline CAPSII & 10,1 & $\begin{array}{l}4,65- \\
21,91\end{array}$ & $<0,001$ & 16,41 & $\begin{array}{l}7,07- \\
38,07\end{array}$ & $<0,001$ \\
\hline CAPSIII & 7,8 & $\begin{array}{c}3,61- \\
16,7\end{array}$ & $<0,001$ & 13,62 & $\begin{array}{l}5,87- \\
31,58\end{array}$ & $<0,001$ \\
\hline \multicolumn{7}{|l|}{ Tipo de Diangósticos } \\
\hline Transtornos Substancias & 1 & - & - & - & - & - \\
\hline Transtornos humor & 2,58 & $\begin{array}{c}1,03- \\
6,46\end{array}$ & 0,04 & - & - & - \\
\hline Transtorno psicóticos & 8,60 & $\begin{array}{l}4,30- \\
17,20\end{array}$ & $<0,001$ & - & - & - \\
\hline Outras morbidades & 5,59 & $\begin{array}{l}2,30- \\
13,60\end{array}$ & $<0,001$ & - & - & - \\
\hline \multicolumn{7}{|l|}{ Número de diagnósticos } \\
\hline 3 & 1 & - & - & - & - & - \\
\hline 2 & 0,88 & $\begin{array}{c}0,34- \\
2,23\end{array}$ & 0,78 & - & - & - \\
\hline 1 & 2,65 & $\begin{array}{c}1,15- \\
6,15\end{array}$ & 0,02 & - & - & - \\
\hline
\end{tabular}

OR - Odds Ratio; IC - intervalo de confiança

de enfermagem de usuários em atendimento intensivo de três CAPS. Desde sua construção e validação, em $2008^{(18)}$, poucas pesquisas o utilizaram e, em contextos de unidades de internação psiquiátrica de hospital geral $^{(10)}$, hospital neuropsiquiátrico ${ }^{(11)}$ e serviço de emergência. ${ }^{(19)}$ Em 2017, sua aplicação em unidades de terapia intensiva (UTI) psiquiátrica, pronto socorro psiquiátrico, enfermaria psiquiátrica e CAPSIII foi recomendada pelo Conselho de Federal de Enfermagem (COFEN), por meio da Resolução no543. (20)

Acreditava-se que o perfil assistencial nos CAPS, com predomínio da categoria discreta no CAPSAD, seguida da intermediária, no CAPSII e III, seria diferente dos encontrados no contexto hospitalar e de emergência; no entanto, as categorias encontradas com frequências elevadas foram semelhantes. ${ }^{(10,11,19)}$
A categoria plena representou a menor parcela em todas as pesquisas ${ }^{(10,11,19)}$, com maior frequência na unidade de internação específica para pessoas em crise. ${ }^{(10)}$ Nas avaliaçôes realizadas nos CAPS encontrou-se apenas um usuário nesta última categoria, implicando em encaminhamento à internação devido ao aumento da demanda de cuidados e, portanto, mudança no regime de tratamento.

Quanto aos diagnósticos identificados, o grupo de transtornos mentais e comportamentais relacionados ao uso de substâncias acarreta prejuízos e sobrecarga aos indivíduos, no entanto, comparado a outros transtornos mentais, não é o mais incapacitante, ficando atrás dos transtornos depressivo e de ansiedade. ${ }^{(17,21)}$ Isto pode explicar sua associação à categoria discreta. Já os transtornos psicóticos, estiveram associados à categoria intermediária/plena, por atingirem desde às funçôes mentais até a rede social e ocupacional dos usuários podendo gerar maior demanda de cuidados. ${ }^{(22,23)}$

Ainda, os transtornos psicóticos foram prevalentes nos CAPSII e III, resultado similar ao de outro estudo. ${ }^{(13)}$ Estes locais constituem-se em serviços de referência à população com transtornos mentais graves, oferecendo apoio e possibilidade de reabilitação em situações de prejuízo cognitivo, dificuldades de reinserção na sociedade, além do sofrimento com o estigma. ${ }^{(17)}$

A presença de mais de uma hipótese diagnóstica em alguns usuários remeteu a situação de que há oscilaçôes para fechar um quadro clínico, principalmente em psiquiatria. $\mathrm{O}$ processo da avaliação diagnóstica demanda tempo, em alguns casos meses ou até anos, sendo natural ocorrer mudanças e associaçôes diagnósticas. ${ }^{(24)}$ Presumia-se que o número de diagnósticos acompanharia a demanda de cuidados, contudo, apresentou-se inverso à classificação das necessidades de atenção de enfermagem. Não se pode concluir que o usuário com um diagnóstico seria um caso de longa data e, portanto, crônico, necessitando de maiores cuidados, pois não foi possível dispor dos dados do tempo de transtorno mental.

Esperava-se, ainda, que as demandas de atenção de enfermagem elevadas correspondessem ao público acima de 50 anos, já que o aumento da idade 
acompanha a necessidade de atenção. ${ }^{(25)}$ Entretanto, as categorias intermediária/plena mostraram-se associadas à faixa etária de 40 a 49 anos. Pesquisa sobre a carga dos transtornos mentais ${ }^{(17)}$ identificou valores mais elevados nesta faixa etária, tanto na esquizofrenia, como no uso/abuso de álcool. Já a categoria discreta esteve associada à faixa etária de 30 a 39 anos, sendo possível inferir que há menor prejuízo na autonomia.

A área de cuidados expressão do pensamento, que avalia a sensopercepçáo e a resposta às solicitaçôes recebeu maior pontuação no CAPSAD, provavelmente por englobar quadros de intoxicação ou síndrome de abstinência de substâncias psicoativas, que podem desencadear sintomas psicóticos, como delírios e alucinações. ${ }^{(26)}$ Muitas vezes esses sinais cessam após o período de horas, no entanto, tem a possibilidade de tornar-se uma condição patológica. ${ }^{(27)}$

Outra área de cuidados, queixas e problemas somáticos, com maior pontuação no CAPSII, está relacionada ao tratamento e efeitos colaterais dos medicamentos. No caso do diagnóstico predominante nesse serviço, transtornos psicóticos, a enfermagem deve atentar-se aos sintomas extrapiramidais como consequência da impregnação de antipsicóticos típicos. ${ }^{(28)}$ Além de desconfortáveis ao indivíduo, a intoxicação pelo uso de antipsicóticos pode levar a um quadro chamado de síndrome neuroléptica maligna, que se não detectado precocemente gera muitas complicaçóes, dentre elas, insuficiência respiratória aguda, lesão renal aguda e sepse. ${ }^{(29)}$ Ainda, nessa área de cuidado, os sinais de cronicidade da doença também são avaliados, e neste caso, podem ser pontuadas pelo prejuízo cognitivo e social gerado por esses transtornos; um exemplo clássico é a esquizofrenia. ${ }^{(30)}$

No CAPSIII a área de cuidado de destaque, sinais vitais e outros controles, tem enfoque na necessidade de um cuidado sistematizado direcionado à sintomatologia de morbidades. Assim como no CAPSII houve o predomínio de transtornos psicóticos, cuja base do tratamento são os antipsicóticos.

(28) Mesmo a nova geração destes medicamentos - os atípicos - pode gerar complicaçôes, como a síndrome metabólica, demandando atenção profissional.
Assim, a alta pontuação nessa área de cuidado pode estar relacionada ao seu uso e ao consequente maior risco para desenvolvimento de doenças como hipertensão arterial sistêmica, obesidade e diabetes mellitus tipo II. ${ }^{(31)}$

Os dois fatores preditores identificados para maior demanda de atenção de enfermagem foram relacionados a ser usuário de CAPSII (16,4 vezes maior), CAPSIII $(13,6)$, e, do sexo masculino $(3,8)$. Ambos CAPS tiveram predomínio de transtorno psicótico. O risco no CAPSII foi 2,8 vezes maior que no III, provavelmente, por ter apresentado maiores frequências na faixa etária (40-49 anos) e no sexo (masculino) associados à maior necessidade de cuidados. A respeito do sexo masculino apresentar-se como um fator de risco, pode estar relacionado à questão cultural masculina de buscar atendimento somente ao surgimento de doenças ou em caso de urgência, além da percepção mais positiva sobre sua saúde do que o sexo feminino. ${ }^{(32)}$ Portanto quando procuram ou são levados aos serviços, os homens podem apresentar a doença em um quadro mais avançado. ${ }^{(33)}$

Os CAPS constituem o modelo de atenção referência do Sistema Único de Saúde (SUS) na Área de Saúde Mental e Psiquiatria, no Brasil, sendo essa pesquisa o primeiro passo para entender como se dá a demanda de cuidados de enfermagem, neste cenário de prática. Assim, espera-se que os achados contribuam e estimulem outras investigaçóes para o aprofundamento nesta temática e a ampliação do olhar da enfermagem para sua assistência.

\section{Conclusão}

As necessidades de atenção de enfermagem de usuários em atendimento intensivo foram predominantes na categoria discreta no CAPSAD e intermediária nos CAPSII e CAPSIII. Identificouse diferenças entre as modalidades no que se refere a sexo, diagnóstico principal e categorias de cuidados. Por fim, os fatores preditores para maior necessidade de atenção de enfermagem constituíram-se em ser usuário dos CAPSII e III, e do sexo masculino. 


\section{Agradecimentos}

O presente trabalho foi realizado com apoio da Coordenação de Aperfeiçoamento de Pessoal de Nível Superior - Brasil (CAPES) - Código de Financiamento 001.

\section{Colaborações}

Pedroso TG contribuiu com a concepção e desenho da pesquisa, obtenção, análise e interpretação dos dados, redação, revisão crítica do manuscrito e aprovação da versão final a ser publicada. Pedrão LJ colaborou com a concepção e desenho da pesquisa, análise dos dados, revisão crítica do manuscrito e aprovação da versão final a ser publicada. Perroca MG cooperou com a concepção e desenho da pesquisa, análise e interpretação dos dados, redação, revisão crítica do manuscrito e aprovação da versão final a ser publicada.

\section{Referências}

1. Amarante $P$, Torre EH. "De volta à cidade, sr. cidadão!" - reforma psiquiátrica e participação social: do isolamento institucional ao movimento antimanicomial. Rev Adm Pública. 2018;52(6):1090 107. https://doi.org/10.1590/0034-761220170130.

2. Brasil. Ministério da Saúde. Secretaria de Atenção à Saúde. Departamento de Atenção Especializada e Temática. Centros de atenção psicossocial e unidades de acolhimento como lugares da atenção psicossocial nos territórios: orientações para elaboração de projetos de construção, reforma e ampliação de CAPS e de UA. Brasília (DF): Ministério da Saúde; 2015. [citado 2020 Fev 18]. Disponível em: http://bvsms.saude.gov.br/bvs/publicacoes/centros_atencao_ psicossocial_unidades_acolhimento.pdf.

3. Pinho ES, Souza AC, Esperidiao E. [Working processes of professionals at Psychosocial Care Centers (CAPS): an integrative review]. Ciênc Saúde Coletiva. 2018;23(1):141-152. Portuguese.

4. Pinto DM, Jorge MS, Pinto AG, Vasconcelos MG, Cavalcante CM, Flores $A Z$, et al. Projeto terapêutico singular na produção do cuidado integral: uma construção coletiva. Texto Contexto Enferm. 2011;20(3):493502.

5. Carey TA. Beyond patient-centered care: enhancing the patient experience in mental health services through patient-perspective care. Patient Exp J. 2016;3(2):46-9.

6. Gabrielsson S, Sävenstedt S, Zingmark K. Person-centred care: clarifying the concept in the context of inpatient psychiatry. Scand J Caring Sci. 2015;29(3):555-62.

7. Gasky L, Coventry P. Person-centred mental health care: the challenge of implementation. Epidemiol Psychiatr Sci. 2012;21,139-144.
8.Bittencourt MN, Marques MID, Barroso TM [Contributions of nursing theories in the practice of the mental health promotion]. Rev Enf Ref. 2018;4(18):125-32. Portuguese.

9. Borges CA, Vasconcelos CR, Oselame GB, Dutra DA. [New profile of nurses front of the Psychosocial Care Center]. Rev Med Saude Brasilia. 2016;5(2):217-33. Portuguese.

10. de Oliveira RP, Laus AM. Caracterização de pacientes de unidade de internação psiquiátrica, segundo grau de dependência do cuidado de enfermagem. Rev Esc Enferm USP. 2011;45(5):1164-70

11. Mukai HA, Jericó MC, Perroca MG. Care needs and workload required by institutionalised psychiatric patients. Rev Lat Am Enfermagem. 2013 Feb;21(1):340-7

12. Clementino FS, Flávia Gomes Silva FG, Miranda FA, Queiróz DT, Pessoa Júnior JM, Marcolino EC, et al. [Perception of users about the care at the Psychosocial Care Center III]. Rev Enferm UFSM. 2017];7(3): 464476. Portuguese.

13. Trevisan ER, Castro SS. [Profile of the psychosocial care center users: an integrative review]. Rev Baiana Saúde Pública. 2017; 41(4):9941012. Portuguese.

14. Peixoto FM, Silva KV, Carvalho IL, Ramos AG, Silva IL, Lacerda GM, et al. Perfil Epidemiológico de Usuários de um Centro de Atenção Psicossocial em Pernambuco, Brasil. J Health Sci. 2017;19(2):114-9.

15. Silva e Silva DL, Torrezan MB, Costa JV, Garcia AP, Toledo VP. [Profile sociodemographic and epidemiologic of users of a psychosocial care center alcohol and drugs]. Enferm Atenção Saúde. 2017;6(1):67-79. Portuguese.

16. Brasil. Ministério da Saúde. Secretaria de Atenção à Saúde. Departamento de Ações Programáticas Estratégicas. Saúde Mental no SUS: Os Centros de Atenção Psicossocial. Brasília (DF): Ministério da Saúde; 2004 [citado 2020 Fev 19]. Available from: http://www.ccs. saude.gov.br/saude_mental/pdf/sm_sus.pdf.

17. Bonadiman CS, Passos VM, Mooney M, Naghavi M, Melo AP. A carga dos transtornos mentais e decorrentes do uso de substâncias psicoativas no Brasil: Estudo de Carga Global de Doença, 1990 e 2015. Rev Bras Epidemiol. 2017 May;20(20 Suppl 01):191-204.

18. Martins PA, Arantes EC, Forcella HT. [Patient classification system in psychiatric nursing: clinical validation]. Rev Esc Enferm USP. 2008;42(2):233-41.

19. Gatti YA, Lopes MC, Costa AF, Campanharo CR, Okuno MF, Batista RE. Classificação do nível de dependência dos pacientes psiquiátricos no serviço de emergência. Cienc Cuid Saude. 2019;18(4):e42151.

20. Conselho Federal de Enfermagem (COFEN). Resolução n543 de 18 de abril de 2017. Atualiza e estabelece parâmetros para 0 dimensionamento do quadro de profissionais de enfermagem nos serviços/locais em que são realizadas atividades de enfermagem. Brasília (DF): COFEN; 2017. [citado 2020 Fev 19]. Disponível em: http://www.cofen.gov.br/resolucao-cofen-5432017_51440.html

21. Vos T, Allen C, Arora M, Barber RM, Bhutta ZA, Brown A, et al.; GBD 2015 Disease and Injury Incidence and Prevalence Collaborators. Global, regional, and national incidence, prevalence, and years lived with disability for 310 diseases and injuries, 1990-2015: a systematic analysis for the Global Burden of Disease Study 2015. Lancet. 2016;388(10053):1545-602.

22. Freitas PH, Pinto JA, Nunes FD, Silva e Souza AR, Machado RM. Esquizofrenia refratária: qualidade de vida e fatores associados. Acta Paul Enferm. 2016;29(1):60-8.

23. Bellier-Teichmann T, Golay P, Bonsack C, Pomini V. Patients' needs for care in public mental health: Unity and Diversity of Self-Assessed Needs for Care. Front Public Health. 2016;4:22. 
24. Leitão IB, Figueiredo DD, Marbach MA, Martins KS. Caracterização dos Transtornos Psiquiátricos Diagnosticados no CAPS I, em Jaguaré, ES, no período de janeiro a outubro de 2014. Rev Psicol Saúde. 2017;9(1):19-35. https://doi.org/10.20435/pssa. v9i1.430.

25. Fonseca C, Ferreira F, Ramos A, Lopes M, Santos V. Study of care needs of people aged 65 and over, proposed intervention of rehabilitation nursing. J Aging Innovation. 2017;6(2):32-42.

26. Da Silva ER, Zerwes Ferreira AC, De Oliveira Borba L, Puchalski Kalinke L, Nimtz MA, Alves Maftum M. Impacto das drogas na saúde física e mental de dependentes químicos. Ciênc Cuid Saúde. 2016;15(1):101-8.

27. Degenhardt L, Saha S, Lim CC, Aguilar-Gaxiola S, Al-Hamzawi A, Alonso J, et al.; WHO World Mental Health Survey Collaborators. The associations between psychotic experiences and substance use and substance use disorders: findings from the World Health Organization World Mental Health surveys. Addiction. 2018;113(5):924-34.
28. Rasmussen SA, Rosebush PI, Mazurek MF. The Relationship between early haloperidol response and associated extrapyramidal side effects. J Clin Psychopharmacol. 2017;37(1):8-12.

29. Modi S, Dharaiya D, Schultz L, Varelas P. Neuroleptic Malignant Syndrome: Complications, Outcomes, and Mortality. Neurocrit Care. 2016;24(1):97-103.

30. Singh S, Khushu S, Kumar P, Goyal S, Bhatia T, Deshpande SN. Evidence for regional hippocampal damage in patients with schizophrenia. Neuroradiology. 2018;60(2):199-205

31. Hirsch L, Patten SB, Bresee L, Jette N, Pringsheim T. Second-generation antipsychotics and metabolic side-effects: canadian population-based study. BJPsych Open. 2018;4(4):256-61.

32. Moura EC, Gomes R, Pereira GM. Percepções sobre a saúde dos homens numa perspectiva relacional de gênero, Brasil, 2014. Cien Saude Colet. 2017;22(1):291-300.

33. Arruda GO, Mathias TA, Marcon SS. Prevalência e fatores associados à utilização de serviços públicos de saúde por homens adultos. Cien Saude Colet. 2017;22(1):279-90. 